\title{
Legislación informática y protección de datos en Colombia, comparada con otros países
}

\author{
María Fernanda Chaparro Ronderos ${ }^{1}$
}

Recibido: Agosto 22 de 2014 Aprobado: Diciembre 10 de 2014

\section{Resumen:}

Actualmente la información de las empresas y las personas tiende a ser almacenada en bases de datos electrónicas, lo cual ha provocado la aparición de diferentes formas de delitos informáticos derivados de la utilización de la información con fines lucrativos o maliciosos, o la alteración de la misma. Para tratar esos delitos se han desarrollado diferentes normativas gubernamentales, como la ley orgánica de protección de datos personales en España, o la ley 1581 de 2012 sobre protección de datos personales en Colombia. Para el presente artículo se realizó una revisión bibliográfica referente al avance en la legislación de protección de datos personales y delitos informáticos en Colombia, Estados Unidos y España, éstos últimos usados como referentes en Colombia para la creación de la legislación en esta materia.

Palabras clave: Seguridad informática, Legislación, Datos personales, Delitos informáticos.

\section{Abstract:}

Currently the information of companies and people tend to be stored in electronic databases, which has led to the emergence of different forms of computer crimes such as the use of the information for profit or malicious treatment, or its alteration. To avoid them, it have been developed different governmental regulations, i.e. the organic law protecting personal data in Spain, or the law 1581 of 2012 for protection of personal data in Colombia. For this paper it was conducted a bibliographic reference review to the progress of legislation regard the protection of personal data and computer crimes in Colombia, the U.S. and Spain, both latters used as references in Colombia for the creation of the legislation in this area.

Keywords: Information security, Legislation, Privacy data, Informatics crimes.

\footnotetext{
1 Ingeniera electrónica, especialista en Seguridad Fisica y de la Información, docente del Programa de Tecnología en Electrónica - UNIMINUTO Sede Principal.
} 


\section{INTRODUCCIÓN}

La información referente a datos privados de las personas y de las empresas que se maneja hoy en día en bases de datos, Call Centers y oficinas estatales, entre otras entidades, ha hecho evolucionar rápidamente el desarrollo de normativas tales como el Real Decreto 1720/2007 y la Ley orgánica 15 de 1999 en España, o la Ley 1581/2012 en Colombia; esto con el fin de propender por la salvaguarda de dichos datos, en especial en cuanto a la información almacenada de forma digital (Pagallo, 2012) (Chen Mok, 2010), en los diferentes escenarios que se presentan, puesto que con el advenimiento de la Internet y los sistemas informáticos abiertos, también han aparecido delincuentes informáticos interesados en recoger los datos almacenados de forma electrónica para utilizarlos con fines lucrativos o maliciosos, o para impedir su utilización. Es por esto que cobra especial relevancia el conocimiento de la Legislación de Protección de Datos Personales y Delitos Informáticos con que se cuenta (Cano, 2012) actualmente en Colombia (Chen Mok, 2010), que es de reciente creación si se compara con los avances alcanzados en este tema en Estados Unidos y España durante los últimos años, sabiendo que estos países se han constituido en referente para Colombia. A partir de este marco se puede realizar una reflexión sobre las implicaciones, objetivos y alcances de la norma para el manejo de la información a nivel informático en nuestro país, y cómo la generación de dichas normativas puede llegar a afectar o beneficiar el uso y la confianza en las Tecnologías de la Información y las Comunicaciones - TIC (Chen Mok, 2010) (Pérez, Rodríguez, Flórez, \& Martínez, 2010) en el entorno colombiano.

\section{METODOLOGÍA}

El método planteado para esta revisión bibliográfica fue la búsqueda en las siguientes bases de datos: IEEE Xplore, Scopus, Google Scholar y Dialnet, utilizando como filtro las palabras seguridad informática y legislación. Se seleccionaron artículos publicados entre 2009 y junio de 2014, teniendo en cuenta que es en los últimos años cuando se ha observado el avance en materia de legislación contra los delitos informáticos en la mayoría de países de América Latina, entre ellos Colombia.

En la base de datos IEEE Xplore se utilizó el filtro tipo de documento en la opción "journals and magazines". Bajo estos criterios se encontraron siete (7) artículos de los cuales tres (3) tienen relación directa con la seguridad informática, la legislación y los datos personales en Estados Unidos.

En la base de datos Scopus se buscó bajo el siguiente criterio: "information security and legislation", utilizando los filtros de año descritos anteriormente. Adicionalmente se aplicó el filtro "subject área" para los términos "computer science, engineer, social sciences" obteniendo 133 artículos de los cuales siete (7) son de relevancia para la revisión a realizar.

Otra base de datos consultada fue Google Scholar centrando la revisión en el uso de los filtros de tiempo (2009-2014) y como tipo de documento artículo, todos relacionados con Colombia, Estados Unidos y España. Se encontraron 11500 resultados, de los cuales se revisaron 250, encontrando seis (6) artículos y una tesis de grado vinculadas directamente con la seguridad informática y los delitos informáticos en los países de interés para la revisión.

Por último se hizo una búsqueda en Dialnet, obteniendo nueve (9) artículos de los cuales tan solo dos (2) se encuentran relacionados específicamente con el vector de búsqueda dado.

De cada artículo seleccionado se extrajeron los datos necesarios para referenciar y hacer el análisis posterior, tales como: título del artículo, año de publicación, nombre de la revista, país de la revista, nacionalidad de los autores y tipo de institución donde trabajan o realizan investigación los autores. Los datos nombrados anteriormente se obtuvieron de los resumenes o de los artículos completos revisados.

\section{RESULTADOS}

Como primer resultado se presenta la Tabla 1 en donde se observa una síntesis de los artículos encontrados por base de datos, con respecto a la cantidad de publicaciones por año.

Tabla 1: Cantidad de artículos / año en las bases de datos consultadas

\begin{tabular}{|c|c|c|c|c|c|c|c|}
\hline \multirow[t]{2}{*}{ BASES DE DATOS } & \multicolumn{6}{|c|}{ AÑO } & \multirow[t]{2}{*}{ TOTAL } \\
\hline & 2009 & 2010 & 2011 & 2012 & 2013 & 2014 (enero-junio) & \\
\hline IEEE Xplore & 2 & 0 & 1 & 0 & 0 & 0 & 3 \\
\hline
\end{tabular}




\begin{tabular}{|l|c|c|c|c|c|c|c|}
\hline \multicolumn{7}{|c|}{ BASES DE DATOS } & \multicolumn{7}{c|}{ AÑO } & TOTAL \\
\hline SCOPUS & 0 & 0 & 0 & 3 & 1 & 3 & 7 \\
\hline Google Scholar & 0 & 0 & 2 & 3 & 1 & 0 & 6 \\
\hline Dialnet & 0 & 2 & 0 & 0 & 0 & 0 & 2 \\
\hline Búsqueda realizada entre el 15 de junio y el 7 de julio de 2014 Fuente: la autora \\
\hline
\end{tabular}

A partir de los 18 artículos revisados se encontró que los temas tratados son genéricos y van enfocados en gran parte a la exploración de la seguridad de la información en ámbitos comerciales. Se observa que los dos artículos de Dialnet ofrecen información exacta de acuerdo con el vector de búsqueda utilizado; uno de los dos artículos (Mok, 2010) se refiere específicamente a la privacidad de datos y su protección, pero presenta el inconveniente de estar con la normativa actualizada hasta el año 2010, por lo cual no hace referencia a las normas y decretos expedidos entre el año 2010 y el primer semestre de 2014. Además, hace mayor énfasis en el proyecto de Ley 15178 de protección de la persona frente al tratamiento de sus datos personales en Costa Rica, desde la perspectiva del comercio electrónico. En el segundo artículo (Pérez et al., 2010) se hace un análisis conceptual sobre los delitos informáticos y su evolución a través del tiempo, planteado desde la perspectiva de autores tanto nacionales como internacionales, y cómo la ley 1273 de 2009 en Colombia hace frente al ciber crimen tanto desde la perspectiva técnica como desde la jurídica, al igual que los diferentes estándares internacionales que sirvieron de base para su creación; sin embargo por su fecha de publicación no tiene en cuenta lo ocurrido en los últimos 4 años, careciendo de un análisis sobre la publicación de la ley 1581 de 2012 y sus repercusiones técnicas y legales en Colombia. De igual manera se encuentra que un artículo ubicado a través de Google Scholar denominado "Protección de datos en Colombia: Avances y retos" (Cano, 2012) cual muestra un resultado parcial de una tesis doctoral en protección de datos personales en Colombia, concerniente a "la seguridad jurídica y el reconocimiento de los derechos fundamentales: habeas data, intimidad, honra y buen nombre, información y libertad informática en el ámbito de las nuevas tecnologías de la información y las comunicaciones y las exigencias internacionales" (Cano, 2012, p. 1), conviertiéndose en referencia para este artículo.

Con respecto al año de publicación, en la Figura 1 se puede ver que existe una media de 3.16 artículos por año, siendo los años 2011, 2012 y enero-junio de 2014

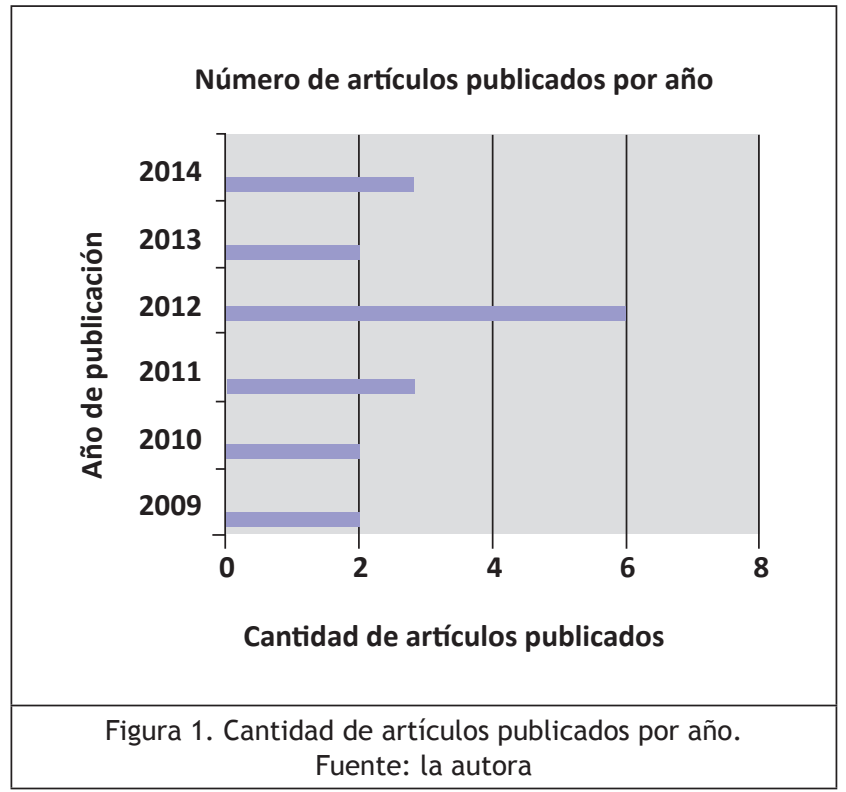

Otro dato a resaltar es que el área de mayor publicación es la de derecho, mientras que en las revistas de ingeniería se nota un gran vacío en publicaciones referentes a este tema.

Con respecto a la institución donde trabajan o realizan investigación los autores, se observa que prácticamente todos (con excepción de un caso), son investigadores de universidades o están realizando trabajos de grado para maestría o doctorado. El único caso que no corresponde a este patrón, es el estudio generado por funcionarios de la CEPAL.

\section{DISCUSIÓN}

A pesar de la gran cantidad de publicaciones que se encuentran con respecto a la seguridad informática (como las hechas por la IEEE, por ejemplo en la revista Engineering Technology o la revista International Journal of Advancements in Computing Technology de los Estados Unidos ), se concluye que en relación con el tema de análisis y discusión de la normativa referente a la protección de los datos personales por parte del área de ingeniería y seguridad, que es la más involucrada en cuanto a la manipulación de esta información, es muy poco lo que se encuentra. (Pérez et al., 2010) especialmente en los capítulos 3 y 4, 
hace un análisis muy detallado sobre la incidencia de la normativa en contra del ciberdelito aprobada en Colombia, pero no se tienen análisis posteriores para el país, ni específicamente relacionados con el impacto generado por la ley 1581 de 2012 en el tratamiento y manejo de los datos personales, las implicaciones jurídicas y técnicas que ha traído la generación e implantación de dicha norma.

En (Mok, 2010) se hace una comparación de las diferentes legislaciones informáticas y su avance en América, pero no se encuentra un análisis con respecto a otras regiones del mundo (Estados Unidos y España).

En cuanto a los artículos encontrados en IEEE Xplore como (Knights, 2011), se observa la gran preocupación de los autores con respecto a la repercusión de la normativa en la Internet y el comercio electrónico o en la protección de los datos privados de prescripción médica (Kosseim \& El Emam, 2009), pero no se observan claramente comparativas ni posiciones con respecto a la interacción con las normativas de otros países o de otros continentes.

Cabe mencionar que el resultado parcial de la tesis doctoral Protección de datos en Colombia: Avances y retos (Cano, 2012), es el más destacado en este artículo debido a que realiza la revisión de la normativa generada en Colombia a partir de terceros países, desde la ley Estatutaria 1266 de 2008 que regula el derecho de habeas data, hasta la ley estatutaria 1581 de 2012 la cual regula la protección de los datos personales. En el capítulo 2 de este resultado parcial (Cano, 2012, p. 11), es muy interesante encontrar que todo el capítulo refiere las tendencias internacionales y su impacto en Colombia, resaltando la "Directiva 95/46/CE", sobre protección de datos personales y la libre circulación de estos datos adoptada por el Parlamento Europeo, así como la Resolución 4595 del 14 de Diciembre de 1990 de la Asamblea General de la ONU denominada "Principios rectores para la reglamentación de ficheros y datos personales".

\section{CONCLUSIONES}

De la búsqueda realizada se puede concluir que existe interés en el tema de la legislación informática en cuanto a la privacidad de los datos personales, pero durante el trascurso de esta revisión no se encontró un estudio transversal actualizado que involucre las disciplinas de ingeniería y derecho; tampoco se encuentra una comparativa que tenga en cuenta distintos continentes (por ejemplo Europa y
América), a pesar de que un gran porcentaje de las normativas generadas en nuestro continente derivan de leyes y normas expedidas en países como España y Estados Unidos.

Debido a la globalización y a la entrada en vigencia de los diferentes tratados de libre comercio de Colombia con otros países, se hace imperiosa la necesidad de realizar un estudio a fondo sobre las diferentes normativas con respecto a la protección de los datos privados, a fin de minimizar los vacíos jurídicos que pueden permitir a los ciberdelincuentes actuar sin temor al castigo. Así mismo, surge la necesidad de que las personas dedicadas a las TIC en nuestro país, conozcan de manera más profunda los acuerdos, normas y leyes que rigen la protección de los datos privados para dar trámite adecuado a las implementaciones que así lo requieran.

En todos los documentos consultados llama la atención el hecho de que aunque se citan estándares o estudios realizados por entidades norteamericanas, no se hace referencia a ninguna de las leyes generadas por los Estados Unidos en esta materia, ni sus repercusiones e influencia en la normatividad Colombiana y Latinoamericana, lo cual indica que en nuestro país la mayoría de considerandos y referentes vienen de Europa y la Unión Europea, con todas las implicaciones que acarrea, entre otras el tratamiento de la información personal de igual manera por ambas partes, permitiendo a nuestro país cumplir más rápidamente con ciertos requisitos técnicos exigidos para la realización de negocios con la Unión Europea o los países que la integran, pero no así con los Estados Unidos, que es uno de nuestros principales socios comerciales.

Al revisar detalladamente se encuentra que la Ley de Transferibilidad y Responsabilidad del Seguro Sanitario (HIPAA, por sus siglas en inglés y promulgada en los Estados Unidos en 1996) no se toma en cuenta para hacer los análisis y comparativas con normatividad de otros países, puesto que solo hace referencia a datos privados relacionados con el área de la salud. Por otro lado, también se tiene en los Estados Unidos el FACTA, aprobada en 2003 como la Ley Federal de Transacciones Crediticias Justas y Exactas, la cual pretende crear un marco normativo para la protección de los datos personales al realizar transacciones crediticias, pero no va más allá. Por lo tanto se puede ver que las leyes en Estados Unidos en materia de protección de datos personales no son tan fuertes como en Europa, lo cual podría determinar el hecho de que no hayan sido tenidas en cuenta para llevar a cabo las comparativas encontradas. 
Si se estudia a profundidad la ley orgánica de protección de datos de carácter personal de España, se puede observar que esta ley tiene por objeto "garantizar y proteger, en lo que concierne al tratamiento de los datos personales, las libertades públicas y los derechos fundamentales de las personas físicas, y especialmente de su honor e intimidad personal y familiar." («LOPD_consolidada.pdf», s. f., Capítulo 1) , lo cual concuerda con lo dicho por la normativa vigente en Colombia, la cual señala que:

La ley tiene por objeto desarrollar el derecho constitucional que tienen todas las personas a conocer, actualizar y rectificar las informaciones que se hayan recogido sobre ellas en bases de datos o archivos, y los demás derechos, libertades y garantías constitucionales a que se refiere el artículo 15 de la Constitución Política; así como el derecho a la información consagrado en el artículo 20 de la misma («LEY 1581 DEL 17 DE OCTUBRE DE 2012.pdf», s. f., p. 1). Esto da una idea de el por qué todos los estudios y artículos que realizan comparativas con relación a la normativa en este campo, se encuentran relacionadas con la legislación expedida en Europa y específicamente en España, ya que tanto Europa como América Latina buscan proteger a la persona en sus derechos fundamentales, antes que a las empresas, dándole así a la Ley un carácter más humanístico.

Aunque en Colombia se promulgó la ley 1712 de marzo de 2014, por la cual "se crea la ley de transparencia y derecho de acceso a la información pública nacional y otras disposiciones", en las bases de datos consultadas no se localizaron referentes al respecto, aunque el gobierno colombiano ha generado iniciativas para su promulgación, revisión o comentarios, como la disponible en la página http: / / www.urnadecristal.gov.co/gestion-gobierno/dejatus-comentarios-al-decreto-que-reglamenta-ley-detransparencia, desde el mes de Septiembre de 2014.

Con respecto al interrogante: ¿qué análisis comparativos se han desarrollado sobre la normatividad relacionada?, se puede decir que en las bases de datos consultadas no se registran análisis profundos y rigurosos de estas normativas, que permitan hacerse una idea del nivel de avance que se tiene en nuestro país actualmente. Por lo tanto, sería muy conveniente adelantar esta clase de estudios y publicar sus resultados en revistas donde las disciplinas de ingeniería, tanto como las de derecho, puedan encontrar un referente teórico más completo.

\section{REFERENCIAS BIBLIOGRÁFICAS}

1. Beckers, K., Côté, I., Faßbender, S., Heisel, M., \& Hofbauer, S. (2013). A pattern-based method for establishing a cloud-specific information security management system: Establishing information security management systems for clouds considering security, privacy, and legal compliance. Requirements Engineering, 18(4), 343-395. doi:10.1007/s00766-013-0174-7

2. Bertot, J. C., Caidi, N., Gorham, U., Jaeger, P. T., \& Shilton, K. (2012). Ten years later: Information and policy in the aftermath of 9/11. Proceedings of the ASIST Annual Meeting, 49(1). doi:10.1002/meet.14504901072.

3. Briones, E. S. (2007). El sentido de la privacidad, la intimidad y la seguridad en el mundo digital: ámbitos y límites. Eguzkilore: Cuaderno del Instituto Vasco de Criminología, (21), 185-199.

4. Cano, L. G. (2012). PROTECCIÓN DE DATOS EN COLOMBIA, AVANCES Y RETOS. Revista LEBRET, 4(4). Recuperado a partir de http://revistas. ustabuca.edu.co/index.php/LEBRET/article/ view/336.

5. Cheng, F.-C., \& Lai, W.-H. (2012). The observation of regulatory approach within internet activities in the United States. International Journal of Advancements in Computing Technology, 4(15), 421-428. doi:10.4156/ijact.vol4.issue 15.49.

6. Chen Mok, S. (2010). Privacidad y protección de datos: un análisis de legislación comparada. Diálogos Revista Electrónica de Historia, 11(1), 111-152.

7. Goel, S., \& Shawky, H. A. (2014). The impact of federal and state notification laws on security breach announcements. Communications of the Association for Information Systems, 34(1), 37-50.

8. Knights, M. (2011). IT security legislation guide. Engineering Technology, 6(7), 63-65.

9. Kosseim, P., \& El Emam, K. (2009). Privacy Interests in Prescription Data, Part I: Prescriber Privacy. IEEE Security Privacy, 7(1), 72-76. doi: 10.1109/MSP.2009.14. 
10. LEY 1581 DEL 17 DE OCTUBRE DE 2012.pdf. (s. f.). Recuperado a partir de http://wsp.presidencia. gov.co/Normativa / Leyes / Documents/LEY\%20 1581\%20DEL\%2017\%20DE\%200CTUBRE\%20DE\%20 2012.pdf.

11. LOPD_consolidada.pdf. (s. f.). Recuperado a partir de http://www.agpd.es/portalwebAGPD/ canaldocumentacion/legislacion/estatal / common/pdfs/LOPD_consolidada.pdf.

12. Melo, V., \& Hernando, A. (2011). El Derecho Informático y la gestión de la seguridad de la información - Una perspectiva con base en la norma ISO27001. Revista de Derecho, 29(29). Recuperado a partir de http://rcientificas. uninorte.edu.co/index.php/derecho/article/ view/2700.

13. Mok, S. C. (2010). Privacidad y protección de datos: Un análisis de legislación comparada. Diálogos: Revista electrónica de historia, 11(1), 4-.
14. Pagallo, U. (2012). Cracking down on autonomy: Three challenges to design in IT Law. Ethics and Information Technology, 14(4), 319-328. doi: 10. 1007/s10676-012-9295-9.

15. Pérez, J. E. O., Rodríguez, F. R., Flórez, M. E. A., \& Martínez, L. A. D. (2010). Delitos informáticos y entorno jurídico vigente en Colombia. Cuadernos de Contabilidad, 11(28), 41-66.

16. Temperini. (2013). Delitos Informáticos en Latinoamerica: Un estudio de derecho comparado. 1ra. Parte. (p. 12). Presentado en $1^{\circ}$ congreso nacional de ingeniería informática/sistemas de información, Universidad Tecnológica Nacional de Córdoba, Argentina.

17. Winter, J. S. (2014). Surveillance in ubiquitous network societies: Normative conflicts related to the consumer in-store supermarket experience in the context of the Internet of Things. Ethics and Information Technology, 16(1), 27-41. doi: 10.1007/s10676-013-9332-3. 